\title{
Infiltração Peri-Articular de Corticosteróides na Abordagem da Patologia Músculo- Esquelética em Medicina Geral e Familiar: Uma Revisão Sistemática
}

\author{
Periarticular Corticosteroid Injection in the Therapeutic Approach of \\ Musculoskeletal Disease in General Practice: A systematic Review
}

\author{
Irene TRINDADE $\triangle 1$ \\ Acta Med Port 2015 Sep-Oct;28(5):652-662
}

\section{RESUMO}

Introdução: Internacionalmente constata-se o tratamento de patologia músculo-esquelética em cuidados de saúde primários através de técnicas de infiltração peri-articular de corticosteróides. Este artigo procede à análise da evidência existente, com o objectivo de contribuir para a discussão do desenvolvimento dessa prática, ainda diminuta, em Portugal.

Material e Métodos: Análise qualitativa de estudos controlados aleatorizados, custo-efectividade, transversais e coorte restrospectivo, recorrendo ao modelo PRISMA. Fontes de dados: PubMed, Cochrane Library, Essential Evidence Plus. Critérios de elegibilidade: infiltrações de corticosteróides no tratamento de patologia músculo-esquelética e indicação de contexto em cuidados de saúde primários. Resultados: Foram incluídos nove estudos controlados aleatorizados, três estudos de custo-efectividade, três estudos transversais, três estudos descritivos e um estudo coorte retrospectivo. A maioria dos indicadores de sucesso da utilização de infiltrações de corticosteróides por médicos de medicina geral e familiar mostrou eficácia a curto prazo; a longo prazo não mostrou superioridade comparativamente às intervenções alternativas. O balanço entre os encargos económicos e o aumento da qualidade de vida é favorável à utilização desta técnica em cuidados de saúde primários. Todos os onze estudos que avaliaram a segurança registaram efeitos adversos ligeiros e nenhum grave.

Discussão: O desempenho das técnicas de infiltração peri-articular de corticosteróides permite figurá-las como opção terapêutica no tratamento de patologia músculo-esquelética nos cuidados de saúde primários Portugueses. Importa incentivar estudos, nomeadamente a nível nacional, que suportem tanto os indicadores de eficácia, como de segurança desta técnica.

Conclusão: A infiltração peri-articular de corticosteróides constitui uma opção relativamente eficaz, segura e com potencial de melhoria da prestação de cuidados de saúde primários Portugueses de uma forma economicamente sustentável.

Palavras-chave: Corticosteróides; Cuidados de Saúde Primários; Infiltrações; Medicina Geral e Familiar.

\section{ABSTRACT}

Introduction: Periarticular corticosteroid injection utilization by General Practitioners treating musculoskeletal disease in primary health care system is internationally acknowledged. This article aims at analyzing this evidence and contributing to the discussion of a potential development of the technique in Portugal.

Material and Methods: Qualitative analysis of randomized control trials, cost-effectiveness studies, cross-sectional studies, retrospective cohort studies and observational studies, using PRISMA model. Data Sources: PubMed, Cochrane Library and Essential Evidence Plus. Eligibility criteria: corticosteroid injection mention in the treatment of musculoskeletal disease and an element that indicates primary health care context.

Results: Nine randomized control trials, three cost-effectiveness studies, three cross-sectional studies, three descriptive studies and one retrospective cohort study were reviewed. Most success parameters of corticosteroid injections performed by general practitioners showed short term efficacy; at long term they did not outstand other therapeutic options. The balance between the economic burden ascribed to this technique utilization in primary health care context and gain in subject's quality of life is favorable. The eleven studies assessing security registered only minor side effects and no serious ones.

Discussion: The periarticular corticosteroid injection technique performance places it as a therapeutic option treating musculoskeletal disease in Portuguese primary health care. Nevertheless further evidence is required to support its efficacy and safety parameters, namely at national level.

Conclusion: Corticosteroid injection utilization in primary health care constitutes an effective and safe option, with the potential to improve primary health care service rendering in an economically sustainable way.

Keywords: Adrenal Cortex Hormones; General Practice; Injections; Primary Health Care.

\section{INTRODUÇÃO}

Em Portugal as doenças reumáticas têm uma prevalência aproximada de 20 a $30 \%$. São causa de 16 a $23 \%$ das consultas de clínica geral, ocupam o segundo ou terceiro lugar dos encargos decorrentes do consumo de fármacos e constituem a primeira causa de incapacidade temporária. ${ }^{1}$ Os sintomas reumatológicos manifestam-se principalmen- te como lesões do aparelho músculo-esquelético ligadas à actividade profissional. $^{2}$

As infiltrações de corticosteróides em estruturas peri-articulares aliviam a dor, diminuem a inflamação e aumentam a mobilidade. ${ }^{3}$ Para além do controlo da dor, a intervenção precoce tem como objectivo prevenir a cronicidade ou

1. Unidade de Saúde Familiar Descobertas. Agrupamentos de Centros de Saúde Lisboa Ocidental de Oeiras. Oeiras. Portugal.

$\triangle$ Autor correspondente: Irene Trindade. trindade.irene@gmail.com

Recebido: 26 de Dezembro de 2014 - Aceite: 07 de Julho de 2015 | Copyright @ Ordem dos Médicos 2015 
recorrência dos sintomas. ${ }^{4}$

Relativamente à segurança da utilização das infiltrações em cuidados de saúde primários (CSP), os efeitos adversos ligeiros são os mais frequentes e incluem o eritema cutâneo, alterações do ciclo menstrual, hipopigmentação cutânea local e hematomas. A atrofia músculo-tendinosa ou do tecido celular subcutâneo, a rotura tendinosa e a celulite são os efeitos adversos graves mais frequentes. Embora esteja descrito um caso de fasceíte necrotizante fatal, as complicações graves decorrentes das infiltrações de corticosteróides são raras. ${ }^{5}$

Relativamente aos dados disponíveis sobre a utilização das infiltrações de corticosteróides por médicos de medicina geral e familiar (MGF) que exercem a sua actividade profissional em Portugal, encontrou-se apenas um estudo do tipo descritivo. ${ }^{6}$ Desde pelo menos 1986 que existe a prática desta técnica em CSP noutros países. ${ }^{7,8} \mathrm{Um}$ dos exemplos de integração desta técnica como opção terapêutica em CSP decorreu na Irlanda do Norte na década de 1990. O projecto incluiu a formação de médicos de MGF na área das técnicas de infiltração de corticosteróides, incentivo à sua prática e análise do impacto no sistema de saúde em geral. ${ }^{9,10}$ Assim, surgiu o interesse em analisar a evidência disponível sobre a utilização das técnicas de infiltração de corticosteróides executadas pelos médicos de MGF. Deste modo, pretende-se avaliar se a possibilidade de tornar esta técnica uma opção terapêutica nos CSP portugueses poderia contribuir para a melhoria dos cuidados de saúde prestados. Além da análise do desempenho das técnicas será também tida em consideração a informação relativa à segurança da sua utilização em contexto de CSP.

\section{MATERIAL E MÉTODOS}

Para a estruturação desta análise sistemática aplicou-se o modelo PRISMA (Preferred Reporting Items for Systematic Reviews and Meta-analyses) corresponde a um conjunto de itens baseados na evidência considerados como o mínimo indispensável para elaborar uma revisão sistemática ou meta-análise. ${ }^{11}$

A pesquisa bibliográfica foi realizada através de ferramentas electrónicas de pesquisa avançada e não avançada das seguintes fontes de dados: PubMed, Cochrane Library e Essential Evidence Plus. Não foram aplicados limites de idioma nem de data de publicação. Durante a pesquisa aplicaram-se os termos MeSH (Medical Subject Headings): adrenal cortex hormones e injections e primary health care ou general practice. Apesar de não ser considerada um termo $\mathrm{MeSH}$, mas por ser mais frequentemente utilizada em detrimento do termo adrenal cortex hormones, a palavra corticosteroids foi também incluída na pesquisa em substituição do seu termo MeSH. A pesquisa foi realizada em Junho de 2014.

Relativamente aos critérios de elegibilidade, os cinco tipos de estudo aceites para análise foram: estudos controlados aleatorizados (ECA), estudos de custo-efectividade, estudos transversais, estudos descritivos e estudos de coorte retrospectivos. Foram sujeitos a leitura todos os re- sumos dos artigos encontrados cujo tema principal presente no título do artigo ou revista de publicação incluísse infiltrações peri-articulares de corticosteróides ou referência a CSP.

Como critérios de exclusão considerou-se a inexistência de texto integral associado à entrada resultante da pesquisa, intervenção principal executada por outro médico não pertencente à especialidade de MGF, infiltração sem componente de corticosteróide ou tratamento de outra patologia para além do foro músculo-esquelético.

Os estudos encontrados foram agrupados e analisados por tipo de estudo, sendo a conclusão produto da sua análise conjunta.

Utilizou-se o modelo PICO (Population, Intervention, Comparison, Outcome), um método utilizado para facilitar a definição de uma estratégia de pesquisa - durante uma abordagem da literatura baseada na evidência - para listar e definir todas as variáveis procuradas nos vários estudos analisados. A população em estudo é, directamente, a dos indivíduos sujeitos à intervenção principal e, indirectamente, a dos médicos de MGF como executantes da intervenção. A intervenção definiu-se como infiltração peri-articular de corticosteróides por médicos de MGF para o tratamento de patologias músculo-esqueléticas. Analisou-se os elementos comparativos entre o tratamento de patologia músculo-esquelética com infiltrações peri-articulares de corticosteróides e o tratamento alternativo ou grupo de controlo. Por último, avaliaram-se os indicadores de sucesso e segurança das intervenções nesses diferentes grupos.

Para os tipos de estudo analisados, os principais indicadores de melhoria do estado de saúde incluíram a evolução do grau de intensidade da dor, do grau de inca-

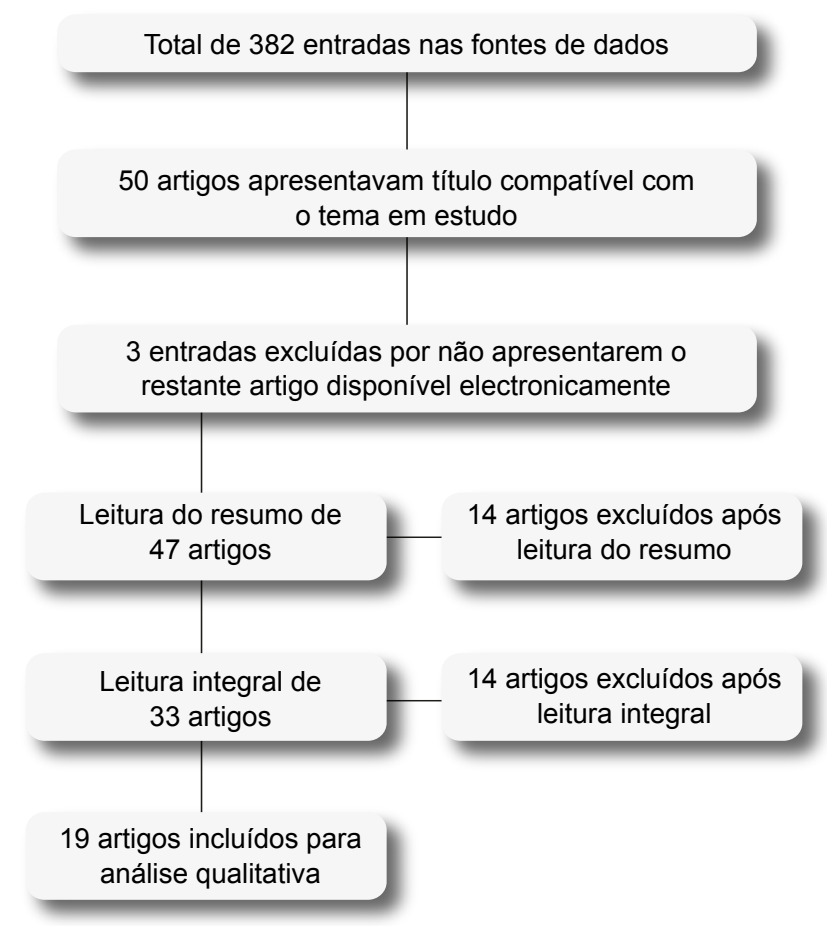

Figura 1 - Diagrama do processo de inclusão dos estudos para revisão 
Tabela 1 - Resumo dos resultados dos estudos aleatorizados controlados incluídos no estudo acerca da avaliação dos parâmetros de sucesso terapêutico das infiltrações de corticosteróides realizadas por médicos de MGF a doentes com patologia músculo-esquelética

\begin{tabular}{l} 
Título do artigo \\
\hline A pragmatic randomized controlled \\
trial of local corticosteroid injection and \\
physiotherapy for the treatment of new \\
episodes of unilateral shoulder pain in \\
primary care ${ }^{12}$
\end{tabular}

Corticosteroid Injections for Greater Trochanteric Pain Syndrome: A Randomized Controlled Trial in Primary Care $^{13}$

\begin{tabular}{|c|c|c|c|}
\hline $\begin{array}{l}\text { Tipo de } \\
\text { estudo }\end{array}$ & NII & NME & $\begin{array}{c}\text { Descrição } \\
\text { diagnóstica }\end{array}$ \\
\hline $\begin{array}{l}\text { Estudo } \\
\text { aleatorizado } \\
\text { multicêntrico } \\
\text { aberto }\end{array}$ & 207 & ND & $\begin{array}{l}\text { Ombro doloroso } \\
\text { unilateral que } \\
\text { agrava com } \\
\text { movimentos } \\
\text { passivos e activos } \\
\text { do MS }\end{array}$ \\
\hline $\begin{array}{l}\text { Estudo } \\
\text { aleatorizado, } \\
\text { multicêntrico, } \\
\text { aberto }\end{array}$ & 120 & 81 & $\begin{array}{l}\text { Dor na face } \\
\text { lateral da anca de } \\
\text { duração superior } \\
\text { a uma semana; } \\
\text { palpação grande } \\
\text { trocânter reproduz } \\
\text { a dor }\end{array}$ \\
\hline
\end{tabular}

Intervenções realizadas Duração do

Infiltração de Avaliação às metilprednisolona no espaço subacromial vs fisioterapia

Analgesia p.o. vs Infiltração de triamcinolona dirigida ao ponto mais doloroso e tecidos adjacentes seguimento

0,6 semana e 6 meses 


\section{Efeitos adversos}

$\begin{array}{lll}\text { Graves } & \text { Ligeiro } \\ \text { ND } & \text { ND }\end{array}$

\section{Principais resultados}

A diferença média na recuperação dos sintomas entre o grupo da fisioterapia e o da infiltração de corticosteróide às seis semanas foi de -0,5 (IC 95\%, -2,1 a 1,2) e aos seis meses de 1,4 (IC 95\%, -0,2 a 3,0). A diferença entre a taxa de sucesso da fisioterapia vs infiltração de corticosteróides foi de $7 \%$ (IC 95\%, 6,8\% a 20,4\%)

Nenhum Número de casos de

evento afrontamentos (12 vs 11), alterações do ciclo menstrual $(5$ vs 3 ), e reacções alérgicas (12 vs 9) para o grupo submetido a tratamento analgésico convencional vs o submetido às infiltrações, respectivamente. Este último grupo apresentou dor superficial no local da infiltração em 26 indivíduos e 1 caso de cada um dos seguintes efeitos adversos: diminuição sensibilidade local, hematoma e tumefacção locais. Dor superficial no local da infiltração em $40 \%$ dos casos.

Nenhum Afrontamentos $(n=9)$, dor no evento local injecção $(n=6)$.
A intensidade da dor em repouso e à mobilização activa diminuiu em ambos os grupos apesar da melhoria ter sido maior no grupo sujeito à infiltração de corticosteróide: diferença ajustada para a dor em repouso foi de $\mathrm{OR}=1,18$ (IC $95 \%, 0,31-2,05)$, para a dor à mobilização activa $\mathrm{OR}=1,30$ (IC 95\%, 0,32 2,29)

Satisfação do doente uma semana pós-intervenção: 16/25 no grupo da infiltração corticosteróides vs 5/25 infiltrações SF $(p<0,001)$; Satisfação aos 12 meses $15 / 25$ infiltração corticosteróides vs $17 / 25$ infiltrações SF $(p=0,322)$
Nenhum Dor duração < 1 dia $(10 \%)$, > evento 1 dia (16\%), dor irradiada ao antebraço $(27 \%)$, afrontamentos (3\%), irritação cutânea local $(5 \%)$, edema e eritema do cotovelo (3\%), alteração da coloração da pele (11\%)

\section{Nenhum Dor local $<1$ dia duração $(\mathrm{n}=$ evento 9 ); $>2$ dias duração $(n=16)$, febre $(n=4)$ irritação cutânea local $(n=1)$; afrontamentos ( $n$ $=9)$, irregularidades menstruais $(n=6)$}

Nenhum evento
Dor local $(n=14)$, afrontamentos $(\mathrm{n}=7)$ sintomas vasovagais $(\mathrm{n}$ $=3$ ), irregularidades menstruais $(n=2)$,
Às seis semanas, existem diferenças significativas a favor da infiltração de corticosteróides para todos os parâmetros de sucesso avaliados $(p<0,0001)$. Fisioterapia apresentou uma taxa de sucesso a 12 meses de $91 \%$, a analgesia p.o. $83 \%$ e a infiltração de corticoide $69 \%$.
Sete semanas após a intervenção a taxa de sucesso para infiltração corticosteróides foi de $77 \%$ vs $46 \%$ para a fisioterapia, diferença de $31 \%$ (IC 95\%, $14 \%$ a $48 \%$ ). Diferenças significativas a favor da infiltração de corticosteróides para todos os parâmetros de sucesso avaliados. Às 36 e 52 semanas pequena variação de parâmetros entre os 2 grupos $(p>0,001)$.
A avaliação a um mês: melhores resultados na melhoria da intensidade dos sintomas no grupo de infiltração de corticosteróides vs infiltração de SF $(p<0,001$, NNT $=3$ ). Taxa de respondedores à infiltração de corticosteróides foi de $91 \%$, desses $49 \%$ apresentaram recorrência dos sintomas durante o seguimento. Os restantes $51 \%$ mostraram deterioramento dos benefícios atingidos a curto prazo durante os 12 meses de seguimento. médicos MGF participantes no estudo, entidade nosológica sujeita à intervenção, intervenções realizadas e principais resultados, incluindo a respectiva validação estatística. Nos estudos de custo-efectividade e de coorte retrospectivo, os parâmetros avaliados foram: o número de indivíduos e de médicos de MGF incluídos no estudo, a entidade nosoló- gica sujeita à intervenção e os principais resultados com validação estatística. Nos estudos transversais, os parâmetros avaliados foram: taxa de resposta aos questionários, número de questionários avaliados, outras especialidades também incluídas no estudo e principais resultados com validação estatística. 
Tabela 1 - Resumo dos resultados dos estudos aleatorizados controlados incluídos no estudo acerca da avaliação dos parâmetros de sucesso terapêutico das infiltrações de corticosteróides realizadas por médicos de MGF a doentes com patologia músculo-esquelética

\begin{tabular}{|c|c|c|c|c|c|c|}
\hline Título do artigo & $\begin{array}{l}\text { Tipo de } \\
\text { estudo }\end{array}$ & NII & NME & $\begin{array}{c}\text { Descrição } \\
\text { diagnóstica }\end{array}$ & $\begin{array}{l}\text { Intervenções } \\
\text { realizadas }\end{array}$ & $\begin{array}{l}\text { Duração do } \\
\text { seguimento }\end{array}$ \\
\hline $\begin{array}{l}\text { Nitroglicerina transdérmica frente } \\
\text { a infiltraciones en las tendinitis del } \\
\text { manguito de rotadores }^{18}\end{array}$ & $\begin{array}{l}\text { Estudo } \\
\text { aletorizado, } \\
\text { controlado, } \\
\text { aberto }\end{array}$ & 48 & ND & $\begin{array}{l}\text { Cumprimento } \\
\text { dos seguintes } \\
\text { critérios: a) Testes } \\
\text { de Impingement, } \\
\text { Jove, Gerbe e } \\
\text { Pate positivos; } \\
\text { b) duração > } \\
6 \text { semanas, c) } \\
\text { ausência de } \\
\text { resposta a AINE }\end{array}$ & $\begin{array}{l}\text { Infiltração local } \\
\text { corticosteróides } \\
\text { vs infiltração de } \\
\text { nitroglicerina, sem } \\
\text { técnica descrita }\end{array}$ & $\begin{array}{l}\text { Avaliação } 0 \text { e } \\
1 \text { semana }\end{array}$ \\
\hline $\begin{array}{l}\text { Randomized controlled trial of local } \\
\text { corticosteroid injections for de Quervain's } \\
\text { tenosynovitis in general practice }{ }^{19}\end{array}$ & $\begin{array}{l}\text { Estudo } \\
\text { aleatorizado } \\
\text { controlado } \\
\text { duplamente } \\
\text { cego }\end{array}$ & 21 & 11 & $\begin{array}{l}\text { Tenosinovite de } \\
\text { Quervain definida } \\
\text { como dor na } \\
\text { estilóide radial } \\
\text { associada quer } \\
\text { a um teste de } \\
\text { Finkelstein positivo } \\
\text { quer a crepitações } \\
\text { palpáveis na } \\
\text { estilóide radial. }\end{array}$ & $\begin{array}{l}\text { Infiltração de } \\
\text { triamcinolona } \\
\text { ao longo da } \\
\text { linha tendinosa, } \\
\text { proximal ou distal } \\
\text { à estilóide radial, } \\
\text { no ponto doloroso } \\
\text { de máxima } \\
\text { intensidade vs SF }\end{array}$ & $\begin{array}{l}\text { Avaliação a } \\
0,1,3,6,12 \\
\text { meses }\end{array}$ \\
\hline $\begin{array}{l}\text { Corticosteroid injection for shoulder } \\
\text { pain: single-blind randomized pilot trial in } \\
\text { primary care }{ }^{20}\end{array}$ & $\begin{array}{l}\text { Estudo } \\
\text { aleatorizado } \\
\text { controlado } \\
\text { em ocultação } \\
\text { simples }\end{array}$ & 19 & ND & $\begin{array}{l}\text { Tendinopatia da } \\
\text { coifa dos rotadores } \\
\text { ou capsulite } \\
\text { adesiva do ombro }\end{array}$ & $\begin{array}{l}\text { Infiltração } \\
\text { no espaço } \\
\text { subacromial de } \\
\text { metilprednisolona } \\
\text { vs lidocaína }\end{array}$ & $\begin{array}{l}\text { Avaliação } \\
\text { às } 4 \text {, e } 12 \\
\text { semanas }\end{array}$ \\
\hline
\end{tabular}

AINE: Anti-inflamatório não esteróide; IC: Intervalo de confiança; MS: Membro superior; ND: Não disponível; NII: Número de indivíduos intervencionados; NME: Número de médicos Medicina Geral e Familiar envolvidos; NNT: Número necessário para tratar; NTG: Nitroglicerina; OR: Odds ratio; p.o.: per os (via oral); SF: Soro fisiológico; vs: versus.

\section{RESULTADOS}

Na Fig. 1 apresenta-se o diagrama do processo de inclusão dos estudos para revisão. Durante a pesquisa realizada foram encontradas 382 entradas no total, contudo apenas 50 artigos referiram os descritores em estudo. Foram excluídas três entradas por não apresentarem o restante artigo disponível electronicamente. Após leitura de 47 resumos foram excluídos 14 artigos, dois por utilizarem artigos originais elegíveis para esta revisão e os restantes 12 com recurso aos critérios de elegibilidade e de exclusão, tendo sido analisados integralmente 33 artigos. Destes últimos, 14 foram excluídos da análise por não cumprirem os critérios de elegibilidade ou por apresentarem critérios de exclusão. Não foram identificados casos de plágio ou fraude entre os artigos analisados. Os 19 artigos incluídos na presente revisão apresentam datas de publicação compreendidas entre 1997 e 2013.

Atendendo ao vasto espectro de patologias músculo-esqueléticas bem como às suas diferentes abordagens terapêuticas disponíveis, a variabilidade entre os diferentes estudos utilizados para o presente artigo verificou-se no que respeita aos desenhos de estudo, às patologias englobadas em cada estudo, à existência de comparação entre a intervenção a analisar (infiltrações) e outras intervenções alternativas, e aos indicadores de saúde avaliados. Considerando que as variáveis encontradas não permitem a sistematização dos resultados, optou-se por fazer a descrição sumária dos estudos e a sua análise qualitativa.
Para efeitos de análise de informação recolhida, subdividiu-se os trabalhos em cinco grupos diferentes: ECA (nove artigos), estudos de custo-efectividade (três artigos), estudos transversais (três artigos), estudos descritivos (três artigos) e um estudo coorte retrospectivo.

Os nove ECA analisados encontram-se sistematizados na Tabela 1. ${ }^{12-20}$ Os indicadores de melhoria do estado de saúde avaliados ao longo do tempo de seguimento incluem a percepção do doente sobre o nível de dor e de incapacidade, e o grau de satisfação antes e após a intervenção. Existiu também variabilidade nos indicadores de melhoria da incapacidade utilizados: amplitude de mobilidade articular, grau de força muscular recuperada pós-infiltração e a aplicação de questionários validados. Em todos os nove estudos os indicadores qualitativos foram mensurados através de escalas de dor ou de incapacidade previamente validadas. Três estudos não apresentaram o número de médicos de MGF envolvidos no estudo. ${ }^{12,18,20} \mathrm{O}$ estudo com maior número de doentes envolvidos $(n=207)$ comparou fisioterapia com infiltrações de corticosteróides no tratamento da síndrome de ombro doloroso com limitação da mobilidade. Às seis semanas a recuperação total foi de $6 \%$ vs $19 \%$, 'alguma melhoria' $72 \%$ vs $54 \%$ e 'agravamento' $6 \%$ vs $8 \%$ para os grupos submetidos a fisioterapia vs infiltração de corticosteróide, respectivamente. Aos seis meses, a recuperação total foi de $24 \%$ vs $18 \%$, 'alguma melhoria' $61 \%$ vs $65 \%$ e 'agravamento' $7 \%$ vs $10 \%$ para os grupos submetidos a fisioterapia vs infiltração de corticosteróide, 


\title{
Efeitos adversos
}

\section{Graves}

Nenhum evento

\section{Ligeiros}

Dor no local da infiltração de corticosteróides em $16 \%$ dos casos. Cefaleia em $62 \%$ dos doentes sujeitos a infiltração de NTG

\section{Principais resultados}

Diferença estatisticamente significativa de 0.58 (IC de $95 \%, 0,35-0,81$ ) a favor da infiltração corticosteróides nos indivíduos que atingiram melhoria total e nos que atingiram melhoria parcial 0,50 (IC de 95\%, 0,27-0,72)
Nenhum evento
Afrontamentos $(n=2)$ e eritema local $(n=6)$.
Avaliação a um mês: efeito benéfico da infiltração de costicosteróides comparativamente ao SF em três dos quatro parâmetros clínicos avaliados $(p=0,015, p=0,047, p=0,031, p=0,112)$, NNT de $2(\mathrm{Cl} 95 \% 1,3)$. Ao longo do seguimento: efeitos benéficos infiltração corticosteróides em duas de três avaliações $(p=0,67, p=0,36, p=0,02)$.

\begin{abstract}
Nenhum ND
evento

A alteração do grau da escala de Oxford para o ombro, na qual maior a pontuação significa maior melhoria clínica, às quatro semanas comparativamente à avaliação inicial foi de $3,9(8,5)$ vs $6,3(10,1)$ para o grupo sujeito a infiltração de corticosteróide comparativamente ao grupo controlo respectivamente. A mesma alteração entre a avaliação inicial e às 12 semanas foi de 4,2 $(9,1)$ vs $8,2(11,3)$ para os mesmos grupos respectivamente.
\end{abstract}

Tabela 2 - Resumo dos resultados dos estudos de custo-efectividade que abordaram a avaliação da qualidade de vida dos doentes com patologia músculo-esquelética sujeita a tratamento com infiltrações de corticosteróides realizadas por médicos de MGF

\begin{tabular}{|c|c|c|c|c|}
\hline Título do artigo & NI & NM & Diagnóstico & Resultados \\
\hline $\begin{array}{l}\text { A cost consequences } \\
\text { analysis of local } \\
\text { corticosteroid injection } \\
\text { and physiotherapy for the } \\
\text { treatment of new episodes } \\
\text { of unilateral shoulder pain } \\
\text { in primary care }{ }^{21}\end{array}$ & 199 & ND & Dor unilateral do ombro & $\begin{array}{l}\text { Aplicação questionários EQ5D }{ }^{\mathrm{TM}} \text { no grupo sujeito à } \\
\text { infiltração de costicosteróides vs grupo fisioterapia, } \\
\text { na primeira avaliação } 0,56 \text { vs } 0,54 \text {, na avaliação a } \\
\text { seis meses } 0,72 \text { vs } 0,75 \text {, respectivamente. Restantes } \\
\text { parâmetros avaliados com diferenças também não } \\
\text { estatisticamente signficativas. }\end{array}$ \\
\hline $\begin{array}{l}\text { Shoulder acute pain in } \\
\text { primary health care: is } \\
\text { retraining GPs effective? } \\
\text { The SAPPHIRE } \\
\text { randomized trial: a cost- } \\
\text { effectiveness analysis }{ }^{22}\end{array}$ & 200 & 155 & $\begin{array}{l}\text { Ombro doloroso (sem } \\
\text { informação diagnóstica } \\
\text { adicional) }\end{array}$ & $\begin{array}{l}\text { O treino de médicos de MGF na área das infiltrações } \\
\text { corticosteróides no período de um ano custou } 298 € \\
\text { adicionais (IC } 95 \%,-334 € \text { a } 932 € \text { ) comparativamente à } \\
\text { não realização dessa formação. O aumento da média de } \\
\text { QALY nos doentes intervencionados foi de } 0.075 \text { (IC } 95 \% \text {, } \\
0,004 \text { a } 0,154) \text {. }\end{array}$ \\
\hline $\begin{array}{l}\text { Cost-effectiveness of } \\
\text { exercise therapy after } \\
\text { corticosteroid injection } \\
\text { for moderate to severe } \\
\text { shoulder pain due to } \\
\text { subacromial impingement } \\
\text { syndrome: a trial based } \\
\text { analysis }\end{array}$ & 107 & ND & $\begin{array}{l}\text { Ombro doloroso (sem } \\
\text { informação diagnóstica } \\
\text { adicional) }\end{array}$ & $\begin{array}{l}\text { Os encargos económicos médios, por doente do Sistema } \\
\text { Nacional de Saúde Britânico ( } 360 € \text { vs } 419 € \text { ) foram } \\
\text { inferiores para o grupo sujeito a infiltração subacromial de } \\
\text { triamcinolona seguida de fisioterapia comparativamente } \\
\text { à fisioterapia isolada, apesar desta diferença não ser } \\
\text { estatisticamente significativa. A melhoria da qualidade de } \\
\text { vida ( } 0,3514 \text { vs } 0,3484 \text { QALYs) foi semelhante para os } \\
\text { dois grupos, respectivamente. }\end{array}$ \\
\hline
\end{tabular}

EQ5D ${ }^{\mathrm{TM}}$ : Questionário EuroQol 5D ${ }^{\mathrm{TM}}$; IC: Intervalo de confiança; MGF: Medicina Geral e Familiar; ND: Não disponível; NI: Número de doentes intervencionados; NM: Número de médicos Medicina Geral e Familiar envolvidos no estudo; QALY: Quality adjusted life years; vs: versus.

respectivamente. ${ }^{12} \mathrm{O}$ estudo de Brinks et al limitado por número insuficiente de indivíduos incluídos no estudo - 120 participantes para uma amostra mínima de 150 - mostrou taxas de recuperação global do síndrome doloroso trocan- térico aos três meses de $55 \%$ para o grupo sujeito a infiltração peri-articular de corticosteróides e de $34 \%$ para o grupo tratado com analgésicos (OR = 2,38; IC 95\%, 1,14 a 5,00$)$. A recuperação aos 12 meses foi de $61 \%$ e $60 \%$ 
Tabela 3 - Resumo dos resultados dos estudos transversais que avaliaram aplicação de questionários a médicos de MGF acerca do tema das infiltrações de corticosteróides no tratamento de patologia músculo-esquelética

\begin{tabular}{lll}
\hline Título do artigo & Especialidades & Taxa resposta \\
\hline $\begin{array}{l}\text { Variations in corticosteroid/anesthetic injections for painful shoulder } \\
\text { conditions: comparisons among orthopedic surgeons, rheumatologists, and } \\
\text { physical medicine and primary-care physicians }{ }^{24}\end{array}$ & MGF & $64 \%$ no total, \\
& MFR & $\begin{array}{l}57 \% \text { para os médicos de MGF } \\
\text { em conjunto com médicos } \\
\text { MFR }\end{array}$ \\
& Reumatologia & Ortopedia
\end{tabular}

Joint and soft tissue injections: a survey of general practitioners ${ }^{25}$

MGF

$70 \%$

Joint and soft tissue injections in the community: questionnaire survey of

MGF

$76 \%$ general practitioners, experiences and attitudes ${ }^{26}$

Especialidades: Especialidade dos médicos envolvidos no estudo; MGF: Medicina Geral e Familiar; MFR: Medicina física e de reabilitação; Questionários: Número de questionários analisados no estudo; Taxa resposta: Taxa de resposta aos questionários enviados; vs: versus.

Tabela 4 - Resumo dos resultados dos estudos descritivos relativos ao tratamento de patologia músculo-esquelética com infiltrações peri-articulares de corticosteróides realizadas pela Medicina Geral e Familiar

\begin{tabular}{|c|c|c|c|c|}
\hline Título do artigo & NI & NM & Diagnósticos & Intervenções \\
\hline $\begin{array}{l}\text { Projecto 'Terapêutica por infiltração local com } \\
\text { corticosteróides nas doenças reumáticas } \\
\text { peri-articulares na USF Marginal' - da ideia à } \\
\text { acção }^{6}\end{array}$ & 74 & 2 & $\begin{array}{l}\text { Ombro doloroso, bursite } \\
\text { trocantérica, doença de } \\
\text { De Quervain, epicondilite, } \\
\text { bursiste anserina e dedo } \\
\text { em gatilho }\end{array}$ & $\begin{array}{l}\text { Infiltração de corticosteróide } \\
\text { peri-articular }\end{array}$ \\
\hline
\end{tabular}

Evaluación de las infiltraciones realizadas en aténcion primaria ${ }^{28}$

Evaluación de las infiltraciones locales con corticóides en un centro de salud ${ }^{27}$

$65 \quad$ ND

\section{Tendinite da coifa dos} rotadores, epicondilite, epitrocleíte, trocânterite, bursiste pré-rotuliana, bursite anserina, tendinite aquílea

138 ND

Infiltração peri-articular de triamcinolona

Infiltração peri-articular de
metilprednisolona

CIT: Certificado de incapacidade temporária; IC: Intervalo de confiança; MGF: Medicina Geral e Familiar; ND: Não disponível; NI: Número de doentes intervencionados; NM: Número de médicos Medicina Geral e Familiar envolvidos no estudo.

(OR = 1,05; IC 95\%, 0,50 a 1,46) para os grupos sujeitos a infiltração e a analgésicos, respectivamente. ${ }^{13}$ No estudo de Peters-Veluthamaningal et al, na avaliação um mês após a intervenção, a frequência de doentes sem novos episódios de dedo em gatilho foi de $70,6 \%$ vs $57,1 \%$ ( $p=$ 0,121 ), recuperação percepcionada pelo doente de $100,0 \%$ vs $92,9 \%(p=0,207)$, para o grupo sujeito a infiltração versus grupo placebo, respectivamente. Avaliando os mesmos parâmetros, 12 meses após a intervenção, os resultados foram: $60,0 \%$ vs $52,9 \%$ ( $p=1000$ ), e $80,0 \%$ vs $100,0 \%$ $(p=0,441)$, para o grupo sujeito a infiltração versus grupo placebo, respectivamente. ${ }^{14}$ Relativo ao tratamento de epicondilite, o estudo de Smidt et al apresenta uma análise conjunta das taxas de sucesso para todas as avaliações realizadas. Nesta, às seis semanas, foi reportado sucesso em $57(92 \%)$ dos doentes no grupo submetido às infiltrações de corticosteróides, $30(47 \%)$ doentes no grupo da fisioterapia e 19 (32\%) no grupo submetido a atitude expectante. Às 52 semanas de seguimento, as taxas de sucesso foram $69 \%$ (43 doentes) para as infiltrações, 91\% (58 doentes) para a fisioterapia e $83 \%$ (49 doentes) para a atitude expectante. ${ }^{15} \mathrm{O}$ estudo de Van der Windt et al avaliou, entre outros parâmetros, a melhoria da restrição dolorosa da mobilidade do ombro por epicondilite através de uma escala Likert de seis pontos, na qual a diferença média (desvio padrão - DP), num intervalo de confiança de $95 \%$, entre o grupo sujeito a infiltração de corticosteróide e o grupo sujeito a fisioterapia foi de 15 (6 a 24) e 11 (1 a 23) às três e 


\section{Questionários}

169 no total conjunto com médicos MFR
44 médicos de MGF em

\section{Resultados}

O resultado da avaliação do conhecimento teórico sobre as características dos fármacos a injectar pelo grupo MGF/MFR foi semelhante ao dos ortopedistas (32\%) e superior ao dos reumatologistas (25\%). Não existe diferença estatisticamente significativa nas doses de corticosteróides utilizadas entres as especialidades estudadas $(p>0,3)$. O Grupo MFR/MGF foi o que menos corticosteróides fluoretados utilizou no tratamento da bainha do bicípite ( $8 \%$ vs $17 \%$ e $37 \%$ ). A percentagem (e número) de médicos em cada grupo que alteraram as suas práticas de infiltração perante um doente diabético foi de $8,6 \%(n=8)$ para os ortopedistas, $13,5 \%(n=37)$ para os médicos de MFR e MGF, e um reumatologista.

$66,4 \%$ dos médicos de MGF realiza as próprias infiltrações, $26,3 \%$ referencia para outro colega de MGF e 7,3\% referencia para os cuidados de saúde secundários. $64,4 \%$ dos médicos envolvidos no estudo tiveram formação na área nos 5 anos anteriores ao estudo.

310
$46 \%$ dos médicos de MGF não praticavam qualquer tipo de infiltração de corticosteróides, desses $83 \%$ já tinham praticado injecções no passado. Um grupo correspondente a $5 \%$ dos clínicos realizou a maioria $(51 \%, 2094 / 4072)$ das injecções realizadas no total em contexto ambulatório. $54 \%$ dos médicos realizaram pelo menos uma infiltração de corticosteróides no ano anterior. $67 \%$ dos médicos respondedores estiveram envolvidos em formação sobre infiltração corticosteróides nos 5 anos anteriores ao estudo.

\section{Efeitos adversos}

Dor nas primeiras 48 horas. Não foram identificados outros efeitos adversos

Um: equimose superficial com resolução expontanea

\section{Resultados}

Dos 74 doentes sujeitos a infiltrações, 48 foram reavaliados aos 11 meses, dos quais $85 \%$ referiram melhoria após a intervenção (52\% 'melhor', 33\% 'muito melhor'; os restantes $15 \%$ referiram 'nem melhor nem pior'.
Ao final de uma semana os indivíduos submetidos a infiltrações de corticosteróides apresentaram uma melhoria estatisticamente significativa tanto da dor 1,4 (IC 95\%, 1 a 1,8) como da incapacidade funcional 0.9 (IC 95\%, 0,7 - 1,1). O mesmo se verificou ao final de um ano: melhoria da dor em 2,3 (IC $95 \%, 1,1-3,5)$ e da impotência funcional em 1,6 (IC 95\%, 1,4 a 1,8); 19 altas de CIT na $1^{\text {a }}$ semana pós tratamento, num universo de 25 indivíduos em absentismo laboral.
Um efeito adverso ligeiro: pigmentação residual da pele no local da infiltração
Os resultados foram avaliados 2 semanas após a intervenção: melhoria dos sintomas em $82,6 \%$ dos casos. Do total, $40,6 \%$ dos doentes referiam melhoria total dos sintomas
52 semanas de seguimento, respectivamente. ${ }^{16}$ No estudo de Peters-Veluthamaningal et al relativamente à eficácia do tratamento da síndrome do túnel cárpico com infiltrações de corticosteróides comparativamente ao controlo (infiltração de soro fisiológico), o primeiro grupo mostrou melhor resposta ao tratamento $(p=0,013)$ e melhor percepção de recuperação pelo doente $(p=0,01)$. O número necessário para tratar (NNT) por forma a atingir uma resposta ao tratamento parcialmente satisfatória ou resolução completa dos sinais e sintomas foi de três (IC 95\%; 1,83 a 9,72). Relativamente à eficácia a longo prazo $51 \%$ (18/35) não reportaram recorrência dos sintomas, sendo que os restantes $49 \%$ (17/35) reportaram. ${ }^{17}$ No estudo de Pons et al os resultados foram avaliados, unicamente, sete dias após a intervenção dirigida ao tratamento da tendinite da coifa dos rotadores. Para os grupos submetidos a infiltração peri-ar- ticular de corticosteróide comparativamente à infiltração de nitroglicerina, $79 \%$ vs $21 \%$ dos doentes obtiveram melhoria total, $12 \%$ vs $21 \%$ melhoria parcial e $8 \%$ vs $58 \%$ não mostraram alteração da condição clínica, respectivamente. ${ }^{18}$ No estudo de Peters-Veluthamaningal et al os resultados do tratamento da tenosinovite De Quervain, avaliados uma semana após a intervenção, mostraram ausência de resposta ao tratamento em $2 / 9$ vs $8 / 12$; resolução completa dos sintomas $6 / 9$ vs $1 / 12(p=0,015)$ no grupo sujeito a infiltração de corticosteróide comparativamente ao grupo controlo. Doze meses após a intervenção, a diferença média do grau de recuperação percepcionado pelo doente e respectivo desvio padrão foi de $1,60(0,73)$ vs $1,03(1,01)$, $p=0,02$, no grupo sujeito a infiltração de corticosteróide comparativamente ao grupo controlo, respectivamente. ${ }^{19}$ No estudo de Holt et al foram aleatorizados 40 doentes 
com tendinopatia ou capsulite adesiva do ombro: um grupo foi submetido a tratamento com infiltração peri-articular de corticosteroide, outro a placebo, sendo o resultado da intervenção avaliado pela Oxford shoulder score. A diferença estimada na alteração desta escala (IC 95\%) comparativamente à avaliação inicial pré-intervenção foi de $-2,4(-8,1$ a $3,4)$ às quatro semanas de seguimento e $-2,9(-9,0$ a 3,2$)$ às 12 semanas. ${ }^{20}$ Em relação à perspectiva do doente, dos três estudos que analisaram a satisfação global do doente, ${ }^{14,15,17}$ um apresenta uma média de satisfação de 3,97 (DP 2,26) numa escala numérica em que zero corresponde a 'não satisfeito' e seis a 'muito satisfeito'; ${ }^{14}$ noutro estudo o índice de satisfação às seis semanas foi de 90\% (DP 16\%) e às 52 semanas de $77 \%$ (DP 23\%) num universo de 62 doentes avaliados. ${ }^{15} \mathrm{O}$ terceiro estudo, já anteriormente referido por limitação do tamanho da amostra, apresenta um NNT de três para atingir satisfação global do doente. ${ }^{17} \mathrm{Um}$ dos nove estudos não apresentava resultados sobre frequência de efeitos adversos. ${ }^{12}$ Não foram registados efeitos adversos graves atribuíveis às infiltrações de corticosteróides apesar da frequência de efeitos adversos ligeiros ter atingido $47 \%$ num dos estudos. ${ }^{15}$ Os efeitos adversos ligeiros atribuídos às infiltrações de corticosteróides, a nível sistémico, incluíram afrontamentos, ${ }^{13-17,19}$ alterações do ciclo menstrual, ${ }^{13,16,17}$ febre, ${ }^{16}$ sintomas vasovagais ${ }^{17}$ e reacções alérgicas; ${ }^{13}$ a nível local registou-se dor superficial, ${ }^{13,15-19}$ irritação cutânea, ${ }^{15}$ alteração da coloração da pele,${ }^{15}$ eritema, ${ }^{15}$ hematoma ${ }^{13}$ e tumefacção. ${ }^{13,15}$

Os três estudos de custo-efectividade analisaram os encargos financeiros e o efeito das infiltrações peri-articulares de corticósteroides na qualidade de vida dos indivíduos com síndrome do ombro doloroso, sendo que nenhum dos estudos fez referência a efeitos adversos. ${ }^{21-23} \mathrm{O}$ estudo de James et al mostrou encargos financeiros totais, por doente, de 100,50€ para o grupo sujeito a infiltrações e 161,59 $€$ para o grupo submetido a fisioterapia, sendo a diferença média, por doente, de 61,08 € (IC 95\%, 22,86 € a 95,92 $€)$. A respectiva análise das alterações de qualidade de vida encontra-se na Tabela $2 .{ }^{21} \mathrm{O}$ estudo de McKenna et al apresenta um ganho de $3992 €$ por cada aumento de um QALY por cada médico de MGF treinado para realizar infiltrações em doentes com patologia músculo-esquelética. ${ }^{22}$ O último estudo apresentado na Tabela 2, de Jowett et al, mostrou que, apesar da diferença não ser estatisticamente significativa, as despesas globais de saúde foram superiores no grupo submetido apenas a fisioterapia (448€ vs 350 $€)$ comparativamente ao grupo submetido a fisioterapia e infiltração de corticosteróide. ${ }^{23}$

A síntese dos estudos transversais que avaliaram aplicação de questionários a médicos de MGF acerca do tema das infiltrações de corticosteróides no tratamento de patologia músculo-esquelética encontra-se na Tabela 3..$^{24-26}$ Um estudo mostra que 164 médicos de MGF $(66,4 \%)$ utilizavam a infiltração peri-articular de corticosteróides na sua prática clínica. ${ }^{25} \mathrm{O}$ mesmo estudo indica que a maioria dos médicos de MGF limita a sua intervenção às patologias do joelho, ombro e cotovelo. Os conhecimentos teóricos dos médicos de MGF acerca das infiltrações de corticosteróides são semelhantes aos avaliados noutras especialidades. ${ }^{24}$ Num dos estudos em que foram avaliadas 244 respostas, as principais barreiras à execução da infiltração de corticosteróides identificadas pelos clínicos foram a incapacidade de manter as aptidões técnicas, a inexistência de treino prático e baixa confiança na execução da técnica. ${ }^{25}$ Noutro estudo, a incapacidade de manter as aptidões técnicas necessárias figurou como principal barreira. ${ }^{26} \mathrm{~A}$ maioria dos médicos de MGF (73\%) treinou em modelos, sendo o treino em doentes reais o que maior nível de confiança proporciona aos clínicos. ${ }^{26}$

Os três estudos do tipo descritivo, um dos quais nacional, abrangeram infiltrações de corticosteróides em diversas áreas anatómicas. O estudo de Gallardo et al e o de Ferreira et al apresentam a taxa de melhoria clínica como resultado principal: $82.6 \%$ e $85 \%$ dos casos, respectivamente. ${ }^{6,27} \mathrm{O}$ estudo de López-Marina et al avaliou o desempenho das infiltrações no tratamento de tendinite da coifa dos rotadores e epicondilite entre outras patologias músculo-esqueléticas, utilizando a escala analógica visual para avaliar o grau de dor e de incapacidade funcional. As médias e intervalos de confiança de $95 \%$ (IC 95\%), para o grau de dor pré-infiltração foi de $8,6(7,4$ a 9,8$)$; uma semana após a infiltração de 1,4 (1 a 1,8); e 2,3 (1,1 a 3,5) um ano depois. As médias e IC 95\% para a aplicação da mesma escala na avaliação da incapacidade funcional foram inicialmente 8,2 (6,7 a 9,7), uma semana após a infiltração $0,9(0,7$ a 1,1$)$ e um ano depois $1,6(1,4$ a 1,8$) \cdot{ }^{28}$ Estes três estudos negam efeitos adversos graves. Quanto aos efeitos ligeiros, um dos estudos apesar de fazer referência à sua ocorrência não os quantifica, ${ }^{6}$ os outros dois trabalhos registaram um efeito adverso ligeiro cada um. ${ }^{27,28}$

O estudo coorte restrospectivo englobou 1188 indivíduos com patologia músculo-esquelética do joelho, anca ou ombro, sem referência ao número de médicos de MGF envolvidos no seu tratamento. Os resultados da pontuação na escala de avaliação ( 0 a 100) em relação à melhoria do estado físico foram de $37,25 \pm 8,39$ vs 42,35 $\pm 9,07$ ( $p=0,0001)$, melhoria do estado mental $52,81 \pm 8,98$ vs $53,54 \pm 8,20(p=0,0001)$, avaliados pré-infiltração e pós-infiltração, respectivamente. Quanto à auto-percepção da dor avaliada pela escala Likert os resultados foram: 3,10 \pm $0,96$ pré-infiltração e 2,36 $\pm 1,21$ ( $p=0,0001)$ pós-infiltração. Este estudo mostrou também uma satisfação global considerada 'satisfatória' ou 'extremamente satisfatória' em relação ao procedimento clínico da infiltração de corticosteroide em 1142 doentes (96\%) e 905 doentes (76\%) reportam 'algum' ou 'muito' benefício com o mesmo. ${ }^{29}$

\section{DISCUSSÃO}

Relativamente à análise dos nove ECA, dos quatro que cumpriram os parâmetros de amostragem necessários, três mostraram resultados estatisticamente significativos a favor do sucesso das infiltrações peri-articulares de corticosteróides a curto prazo comparativamente à intervenção alternativa. ${ }^{14,15,17} \mathrm{O}$ estudo com maior número de doentes 
envolvidos ( $n=207$ ) não mostrou diferença. ${ }^{12}$ Para os mesmos resultados, apenas um estudo mostrou diferença significativa a longo prazo. ${ }^{14}$ No conjunto dos quatro dos nove ECA, que não cumpriram os critérios de amostragem, todos mostraram resultados significativamente positivos a favor da utilização das infiltrações a curto prazo mas nenhum a longo prazo. ${ }^{13,16,19,20} \mathrm{O}$ estudo sem cálculo de amostragem e sem análise dos resultados a longo prazo mostrou superioridade da técnica a curto prazo. ${ }^{18} \mathrm{~A}$ evidência de que os benefícios das infiltrações peri-articulares de corticosteróides não se mantêm a longo prazo já se encontra descrita na literatura. Na maioria destes estudos, a técnica de infiltração foi executada por médicos de outras especialidades para além da MGF. ${ }^{30-32}$ No que respeita às limitações encontradas, os estudos incluídos na presente revisão avaliam infiltrações peri-articulares de corticosteróides no tratamento de entesites de diferentes articulações. O facto da técnica em análise ter sido comparada com diferentes intervenções (fisioterapia, placebo, tratamento analgésico via oral, infiltração de nitroglicerina) também poderá enfraquecer o seu poder de extrapolação.

Da análise dos estudos de custo-efectividade, dois estudos indicam a infiltração de corticosteróides no tratamento da omalgia unilateral como uma opção terapêutica com indicadores de custo-efectividade favoráveis. ${ }^{21,23} \mathrm{Um}$ deles indica esta intervenção como mais económica comparativamente à fisioterapia, mostrando que ambas as opções de tratamento apresentam efeitos benéficos idênticos no âmbito da qualidade de vida. ${ }^{21}$ Outro estudo mostra um balanço positivo entre o investimento na formação de médicos de MGF na área das infiltrações de corticosteróides e a vantagem económica atribuída ao aumento da qualidade de vida dos doentes após essa intervenção. ${ }^{22}$

O estudo coorte restrospectivo mostrou resultados significativamente positivos no que respeita ao alívio da dor e melhoria da qualidade de vida nos doentes com patologia músculo-esquelética submetidos a infiltrações realizadas por médicos de MGF.

Uma das limitações comum aos três estudos transversais baseados em resultados de questionários aplicados a médicos de MGF prende-se com o facto de os questionários serem anónimos. Desta forma, o anonimato não permite averiguar as características dos médicos não-respondedores.

Onze dos 19 estudos analisados incluem os efeitos adversos das infiltrações de corticosteróides nas suas análises. Apesar de nenhum estudo registar efeitos adversos graves, todos descreveram efeitos adversos ligeiros tendo um estudo atingido $47 \%$ e outro $40 \%$ de frequência destes eventos. ${ }^{13,15}$ Estes últimos apresentaram-se não só a nível do local da infiltração como também a nível sistémico. Por outro lado, a infiltração peri-articular de corticosteróides é uma técnica regularmente praticada a nível mundial, considerada por vários autores como uma intervenção relativamente segura. ${ }^{3,33}$ Seria interessante analisar a percepção do doente relativamente ao balanço entre o impacto destes efeitos adversos no seu bem-estar e a satisfação com os resultados obtidos pelo tratamento com infiltração de corticosteróides.

Os três estudos descritivos incluídos nesta revisão apresentam vieses como o eventual efeito placebo da intervenção, amostras de conveniência, entre outras limitações inerentes ao desenho de estudo. Esses estudos não possuem, então, o mesmo poder de inferência que os restantes artigos incluídos nesta revisão.

O único estudo realizado em Portugal sobre o tratamento de patologia músculo-esquelética com infiltrações peri-articulares de corticosteróides em CSP é um estudo do tipo descritivo que mostrou resultados favoráveis. ${ }^{6}$ Introduzir linhas de investigação nesta área, em Portugal, seria uma oportunidade de reunir mais resultados não só de eficácia e sustentabilidade como também de segurança.

De uma forma geral, os indicadores de sucesso da utilização da técnica por médicos de MGF não se mantêm a longo prazo como foi já descrito para a sua utilização por médicos de outras especialidades. Se por um lado, não se registam efeitos adversos graves, por outro verifica-se uma frequência significativa de efeitos adversos ligeiros. Além de serem consideradas financeiramente viáveis, as infiltrações peri-articulares de corticosteróides realizadas em CSP oferecem vantagem da proximidade para o doente e consequentes ganhos socioeconómicos.

Permanecem em aberto linhas de estudo sobre o impacto da descentralização deste tipo de tratamento nos sistemas de saúde, com eventual diminuição das listas de espera para consultas da especialidade de Reumatologia ou Ortopedia. Seria também pertinente estudar a repercussão deste tratamento eficaz a curto prazo no número de altas precoces por Incapacidade Profissional Temporária derivada de patologia músculo-esquelética de origem profissional.

\section{CONCLUSÃO}

O estudo, pese embora a escassez de artigos existentes a nível nacional e internacional, permite retirar algumas conclusões.

Para resultados a curto prazo, as infiltrações peri-articulares de corticosteróides poderão ser realizadas com relativo sucesso e segurança em contexto de CSP, mostrando resultados positivos na satisfação e melhoria da qualidade de vida dos doentes. Pelo contrário, a longo prazo esta técnica não mostra benefícios em relação às intervenções alternativas.

A evidência analisada permite antecipar vantagens na integração das técnicas de infiltração de corticosteróides no conjunto de opções terapêuticas disponíveis para o tratamento de patologia músculo-esquelética nos CSP em Portugal. O maior envolvimento dos médicos de MGF portugueses no tratamento de patologia músculo-esquelética através destas técnicas poderá constituir uma oportunidade de melhorar a qualidade de vida e o controle da dor dos utentes dos CSP em Portugal. Esta iniciativa contribuiria também para o desenvolvimento da prestação de cuidados de saúde de uma forma economicamente sustentável. 


\section{AGRADECIMENTOS}

A Maria Manuel Alves pela disponibilidade e apoio sempre demonstrados.

\section{OBSERVAÇÕES}

A primeira versão deste trabalho foi apresentada e premiada durante o $7^{\circ}$ Curso de Reumatologia Prática em Cuidados de Saúde Primários, Lisboa, 18-19 Setembro 2014.

\section{REFERÊNCIAS}

1. Direcção-Geral da Saúde. Programa Nacional contra as Doenças Reumáticas. Despacho Ministerial de 26/03/2004. Lisboa: DGS; 2004. p. 5

2. Lucas $\mathrm{R}$, Monjardino MT. O estado da reumatologia em Portugal. Lisboa: Observatório Nacional de Doenças Reumáticas - Programa Nacional das Doenças Reumáticas; 2010. p. 85-91.

3. Stephens MB, Beutler Al, O'Connor FG. Musculoskeletal injections: a review of the evidence. Am Fam Physician. 2008;78:971-6.

4. Silva JA. Soft tissue injections. Best Pract Res Clin Rheum. 2005;19:503-27.

5. Brinks A, Koes BW, Volkers AC, Verhaar JA, Bierma-Zeinstra SM Adverse effects of extra-articular corticosteroid injections: a systematic review. BMC Musc Dis. 2010;11:206.

6. Ferreira AR, Carrapiço E, Pimentão JB. Terapêutica por infiltração local com corticosteróides nas doenças reumáticas periarticulares na USF Marginal. Rev Port Med Geral Fam. 2012;28:202-10.

7. Grahame R, Gibson T, Dale E, Anderson JA, Brown R, Higgins $P$, et al. An evaluated programme of rheumatology training for general practitioners. Br J Rheumatol. 1986;25:7-12.

8. Nelson $\mathrm{KH}$, Briner $\mathrm{W} \mathrm{Jr}$, Cummins J. Corticosteroid injection therapy for overuse injuries. Am Fam Physician. 1995;52:1811-6.

9. Helliwell PS, Wright V. Referrals to rheumatology. BMJ. 1991;302:3045 .

10. Lowy A, Brazier J, Fall M, Thomas K, Jones N, Williams BT. Mino surgery by general practitioners under the 1990 contract: effects on hospital workload. BMJ. 1993;307:413-7.

11. Liberati A, Altman D, Tetzlaff J, Mulrow C, Gøtzsche P, Loannidis J, et al. The PRISMA statement for reporting systematic reviews and metaanalyses of studies that evaluate health care interventions: explanation and elaboration. J Clin Epidemiol. 2009;62:1-34.

12. Hay EM, Thomas E, Paterson S, Dziedzic K, Croft PR. A pragmatic randomised controlled trial of local corticosteroid injection and physiotherapy for the treatment of new episodes of unilateral shoulder pain in primary care. Ann Rheum Dis. 2003;62:394-9.

13. Brinks A, van Rijn RM, Willemsen SP, Bohnen AM, Verhaar JA Koes BW, et al. Corticosteroid injections for greater trochanteric pain syndrome: A randomized controlled trial in primary care. Ann Fam Med. 2011;9:226-34.

14. Peters-Veluthamaningal $\mathrm{C}$, Winters JC, Groenier $\mathrm{KH}$, Jong $\mathrm{BM}$. Corticosteroid injections effective for trigger finger in adults in general practice: a double-blinded randomised placebo controlled trial. Ann Rheum Dis. 2008;67:1262-6.

15. Smidt N, van der Windt DA, Assendelft WJ, Devillé WL, Korthas-de Bos IB, Bouter LM. Corticosteroid injections, physiotherapy, or a wait-andsee policy for lateral epicondylitis: a randomised controlled trial. Lancet. 2002;359:657-62.

16. Van der Windt DA, Koes BW, Devillé W, Boeke AJ, de Jong BA, Bouter LM. Effectiveness of corticosteroid injections versus physiotherapy for treatment of painful stiff shoulder in primary care: randomised trial. BMJ. 1998;317:1292-6.

17. Peters-Veluthamaningal C, Winters JC, Groenier H, Meyboom-de Jong B. Randomised controlled trial of local corticosteroid injections for carpal tunnel syndrome in general practice. BMC Fam Pract. 2010;11:54.

\section{CONFLITO DE INTERESSES}

A autora declara não ter nenhum conflito de interesses relativamente ao presente artigo.

\section{FONTES DE FINANCIAMENTO}

O trabalho relatado não foi objecto de qualquer tipo de financiamento externo (incluindo bolsas de investigação).

18. Pons S, Gallardo C, Caballero JC, Martinez T. Nitroglicerina transdérmica frente a infiltraciones en las tendinitis del manguito de rotadores. Aten Primaria. 2001;28:452-6.

19. Peters-Veluthamaningal $\mathrm{C}$, Winters JC, Groenier $\mathrm{KH}$, Meyboom-de Jong B. Randomised controlled trial of local corticosteroid injections for de Quervain's tenosynovitis in general practice. BMC Muskuloskelet Disord. 2009;10:131.

20. Holt T, Mant D, Carr A, Gwilym S, Beard D, Toms C, et al. Corticosteroid injection for shoulder pain: single-blind randomized pilot trial in primary care. Trials. 2013;14:425.

21. James M, Stokes EA, Thomas E, Dziedzick K, Hay EM. A cost consequences analysis of local corticosteroid injection and physiotherapy for the treatment of new episodes of unilateral shoulder pain in primary care. Rheumatology. 2005;44:1447-51.

22. McKenna C, Boike L, Manca A, Adebajo A, Dickson J, Helliwell P, et al. Shoulder acute pain in primary health care: is retraining GPs effective? The SAPPHIRE randomized trial: a cost-effectiveness analysis. Rheumatology. 2009;48:558-63.

23. Jowett S, Crawshaw D, Helliwell PS, Hensor E, Hay E, Conaghan P. Cost-effectiveness of exercise therapy after corticosteroid injection for moderate to severe shoulder pain due to subacromial impingement syndrome: a trial based analysis. Rheumatology. 2013;52:1485-91.

24. Skedros JG, Hunt KJ, Pitts TC. Variations in corticosteroid/anesthetic injections for painful shoulder conditions: comparisons among orthopaedic surgeons, rheumatologists, and physical medicine and primary-care physician. BMC Muskuloskelet Disord. 2007;8:63.

25. Liddell WG, Carmichael CR, McHugh NJ. Joint and soft tissue injections: a survey of general practitioners. Rheumatology. 2005;44:1043-6.

26. Gormley GJ, Corrigan M, Steele WK, Stevenson M, Taggart AJ. Joint and soft tissue injections in the community: questionnaire survey of general practitioners, experiences and attitudes. Ann Rheum Dis. 2003;62:61-4.

27. Gallardo J, Molina A, López B, González J, Bonet F, Aranda C. Assesment of corticoid local injections at a health care center. Aten Primaria. 2000;25:331-4.

28. López-Marina V, Pizarro G, Costa-Bardají N, Mata-Navarro A, VázquezBlanco JR. Evaluación de las infiltraciones realizadas en atención primaria. Aten Primaria. 2005;36:544-9.

29. Eftekhaari TE, Nazarnezhad M, Ghasemzadeh I. Efficacy of musculoskeletal injections by primary care providers in the office: a retrospective cohort study. Int J Gen Med. 2013;6:773-5.

30. Ferreira A. Efectividade das infiltrações de corticosteróides nas doenças reumáticas peri-articulares: uma revisão baseada na evidência. Rev Port Med Geral Fam. 2013;29:38-52.

31. Coombes BK, Bisset L, Vcenzino B. Efficacy and safety of corticosteroid injections and other injections for management of tendinopathy: a systematic review of randomised controlled trials. Lancet. 2010;376:1751-67.

32. Garg N, Perry L, Deodhar A. Intra-articular and soft tissue injections, a systematic review of relative efficacy of various corticosteroids. Clin Rheumatol. 2014;33:1695-706.

33. Dolley P, Martin R. Corticosteroid injections and arthrocentesis. Can Fam Physician. 2002;48:285-92. 
Irene TRINDADE

\section{Infiltração Peri-Articular de Corticosteróides na Abordagem da Patologia Músculo-Esquelética em Medicina Geral e Familiar: Uma Revisão Sistemática}

Acta Med Port 2015:28:652-662

Publicado pela Acta Médica Portuguesa, a Revista Científica da Ordem dos Médicos

Av. Almirante Gago Coutinho, 151

1749-084 Lisboa, Portugal.

Tel: +351218428 215

E-mail: submissao@actamedicaportuguesa.com

www.actamedicaportuguesa.com

ISSN:0870-399X | e-ISSN: 1646-0758

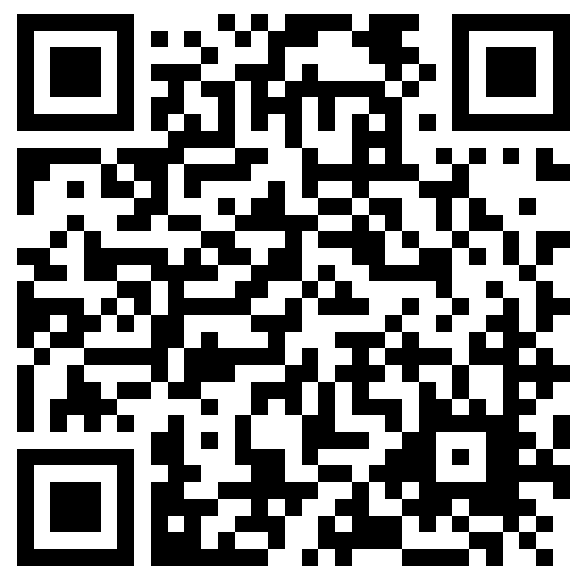

